\title{
The Light Adjustable Lens - A Review
}

\author{
Alejandro Lichtinger
}

Fellow, Cornea, External Diseases and Refractive Surgery, Toronto Western Hospital, University of Toronto, Ontario, Canada

\begin{abstract}
The purpose of this manuscript is to review the design, development and clinical results of the light adjustable lens (LAL). Evolution in techniques, biometry and intraocular lens (IOL) calculation have improved outcomes and increased expectations after cataract surgery. Nevertheless, Imprecise IOL power determinations, pre-existing and surgical-induced astigmatism, and previous corneal refractive surgery continue to limit post-operative uncorrected vision. The LAL was designed to provide a stable, precise correction of refractive errors with a safe, non-invasive post-operative procedure. The concept behind the LAL is based on photochemistry and diffusion. The adjustment occurs when all non-attached solutes equally distribute themselves throughout the optic after irradiation with ultraviolet light that causes the photosensitive macromers to polymerise in the irradiated region. Clinical results have been positive, with the largest series (122 eyes) showing $97 \%$ of patients within 0.25 D of attempted spherical equivalent and $100 \%$ uncorrected vision 20/25 or better. In conclusion, the LAL has demonstrated to be a safe, accurate and reliable method of post-operative, non-surgical correction of residual sphero-cylindrical refractive error.
\end{abstract}

\section{Keywords}

Light adjustable lens, cataract surgery, intraocular lens, intraocular lens calculation

Disclosure: The author has no conflicts of interest to declare.

Received: 28 November 2011 Accepted: 6 January 2012 Citation: European Ophthalmic Review, 2012;6(2):108-11 DOI: 10.17925/EOR.2012.06.02.108 Correspondence: Alejandro Lichtinger, Toronto Western Hospital, University of Toronto 399 Bathurst street, East Wing 6-401, Toronto, Ontario, Canada.

E: drlichtinger@yahoo.com

The concept of an intraocular lens (IOL) that can be adjusted in vivo has been hypothesised and studied since phacoemulsification became the standard of care in cataract surgery ${ }^{1}$ and its potential benefits have been recognised by previous investigators. ${ }^{2-4}$ The first published report of a multicomponent IOL that could be adjusted after implantation came in $1996 .{ }^{5}$ Kraser $^{2}$ patented a three-piece IOL with an inflatable diaphragm in which injection or removal of fluid from the flexible optic is used to post-operatively adjust the IOL power. Eggleston ${ }^{3}$ patented a mechanically adjustable IOL whose power is adjusted by rotation of the optic in a screw guide ${ }^{6}$ and later reported on the possibility of performing the adjustment non-invasively with the use of samarium and cobalt magnets. ${ }^{\text {? }}$

The development of the light adjustable lens (LAL) (Calhoun Vision, Inc, Pasadena, CA) started in 1996 with a collaboration by Daniel M Schwartz from the University of California, San Francisco and Robert Grubbs, Chemistry Professor at the California Institute of Technology (who subsequently won the Nobel prize in Chemistry in 2005), with the objective of creating a lens that was biocompatible, implantable through a small incision and capable of providing a stable, precise correction of myopic, hyperopic and astigmatic refractive errors within a reasonable power range while benefiting from a safe, non-invasive post-operative adjustment procedure. ${ }^{4}$

\section{Design and Development}

Silicone was selected for the LAL technology because of its optical clarity, ability to be folded during insertion and history of safe use in IOLS. The silicone formulation used in the LAL is based upon a silicone matrix system comprised of polymer, resin, crosslinker and platinum catalyst. Formulated into the silicone matrix are a photoreactive macromer, photoinitiator and ultraviolet (UV) absorbers. The photosensitive macromer system was designed to be compatible with the silicone matrix and insoluble in water to prevent leaching into the eye. It also was formulated to remain optically clear upon forming an interpenetrating network within the matrix upon irradiation with UV light. $^{4}$

The LAL is a three-piece silicone lens with blue poly(methyl methacrylate) (PMMA) modified-C haptics. The optic has a diameter of $6 \mathrm{~mm}_{1}^{8}$ a 360-degree square edge on the posterior surface to minimise posterior capsular opacification (PCO) and an enhanced UV protection layer in the posterior aspect to minimise erythropsia from retinal UV exposure. ${ }^{10}$

The basic concept behind the LAL is based on the principles of photochemistry and diffusion. ${ }^{11}$ The optic consists of partially polymerised macromers with an appropriately bonded photo-sensitiser. The adjustment is based upon the principle that all non-attached solutes will equally distribute themselves throughout the lens optic..$^{2}$ Irradiation of the LAL with UV light (wavelength of $365 \mathrm{~nm}$ ) causes the photosensitive macromers to polymerise in the irradiated region. For example, if a central optical zone is irradiated, macromers polymerise in that region alone. Following this polymerisation, macromers in the peripheral, un-irradiated portions of the LAL are highly concentrated relative to the central irradiated zone, where they have been depleted by irradiation. This creates a thermodynamically unstable diffusion gradient, which is corrected over 12 to 15 hours as macromers 
diffuse toward the irradiated region to once again establish uniform concentration throughout the matrix. As the macromers migrate centrally, this portion of the lens swells and refractive power increases to correct hyperopia. ${ }^{4}$

Refractive adjustments are made with the light delivery device (LDD) by delivering light of the selected wavelength, spatial intensity profile, and diameter to produce a predictable change in the power of the LAL. The LDD consists of a UV light source, projection optics, and control interface. An apodised filter is included in the optical assembly to create a specific beam-intensity profile projected onto the implanted lens. ${ }^{4}$ The LDD generates and projects a spatial irradiance pattern onto the LAL using a digital mirror device. The surgeon centres and focuses the treatment beam on the LAL using an alignment reticule while patient alignment is achieved using a fixation target, paracentral to the delivery beam. The LDD is capable of correcting myopia, hyperopia and astigmatism. ${ }^{13}$ Customised multifocal profiles, as well as spherical aberration and other higher-order aberrations treatments can be designed with the LDD. ${ }^{14}$

In practice, the LAL of the appropriate power is implanted into the capsular bag after routine phacoemulsification through a clear cornea incision (3.0 to $3.5 \mathrm{~mm}$ ). After surgery, the patient is instructed to wear UV-light filtering photochromic spectacles (7EYE, Pleasanton, CA) at all times when indoors and outdoors until the final lock-in treatment is completed. Ten to 21 days after implantation, patients have their first refractive adjustment treatment using the LDD to correct post-operative hyperopic, myopic and/or astigmatic refractive errors; in some cases, the nomogram requires two separate adjustments to achieve the desired post-operative refractive correction. After the refractive adjustments are completed, the patient receives two lock-in treatments separated by a minimum of two days. The lock-in treatments are necessary to consume all remaining photoactive macromer in the $L A L$ and stabilise the adjusted lens power.

Adjustment procedures take between 45 and 130 seconds depending on the desired amount of correction.

From a phototoxicity standpoint, the two most important ocular structures that would be affected by treatment of the LAL with UV light are the cornea and the retina. A study by Werner et al., ${ }^{15}$ assessed corneal toxicity in a cat model. Twelve cats had a light beam of $365 \mathrm{~nm}$ with an intensity of $250 \mathrm{~mW} / \mathrm{cm}^{2}$ applied to the central $6.0 \mathrm{~mm}$ of the right cornea for 120 seconds The cats were killed at day one, week one and one and three months after light application (three cats/time point). Their corneas where evaluated for evidence of morphological damage to the endothelial cells by staining with trypan blue and alizarin red and found that the irradiation dose used to treat the LAL was not associated with damage to the endothelial cells. These findings were later confirmed clinically by a study that evaluated the corneal endothelial safety after UV treatment of the LAL in 10 patients by examining endothelial cell counts and morphology before phacoemulsification, one week postoperatively (before any UV treatment) and at six months post lock-in. The study found that the irradiation protocol for adjustment and lock-in of the LAL does not seem to add to the endothelial cell damage caused by the cataract surgery, indicating the protocol is safe to the corneal endothelium.
The same group at the University of Utah performed a retina safety study ${ }^{16}$ in a rabbit model in which they performed phacoemulsification with implantation of the LAL in one eye and a silicone lens with no UV filter in the other eye (to serve as control). The LAL eyes were then irradiated at 1.0, 2.03 .0 and 5.0 times the expected maximum UV irradiation doses and the control eyes at 0.3, 0.6, 1.0 and 2.0. The rabbits were killed a week after the irradiation and their eyes enucleated and processed for histopathology. The 16 eyes implanted with the LAL showed no signs of corneal, anterior segment, or retina toxicity while the three eyes in the control group that received the highest irradiation dose had areas of focal retinal damage. This study showed in an animal model that irradiation doses up to five times the expected maximum irradiation, caused no retinal toxicity in eyes implanted with the LAL.

Before any human trials, extensive in vitro and in vivo studies where performed in regards to cytotoxicity, hydrolytic stability, photostability, optical performance and biocompatibility to ensure the safety and quality of the LAL and the irradiation procedure. ${ }^{4}$ Discussion of the results of these studies is beyond the scope of this review.

\section{Clinical Results}

Chayet et al. reported the first clinical series in the peer-reviewed literature for both pure hyperopic ${ }^{17}$ and myopic ${ }^{11}$ corrections. For the hyperopic series, after biometry, a LAL power was selected to result in a post-operative refractive error of up to +2.00 Dioptres (D) of hyperopia in 14 patients. Adjustments in the range of +0.25 to $+2.0 \mathrm{D}$ were performed and the patients followed for six months. Thirteen of the 14 eyes $(92.9 \%)$ treated were within $0.25 \mathrm{D}$ of the targeted refraction and all adjustments were stable within $0.25 \mathrm{D}$ by last follow up. The same amount of patients were enrolled for the myopia correction study. The refractive adjustments attempted in this study ranged from -0.25 to $-1.50 \mathrm{D}$ of myopia. Ninety-two percent of the eyes treated were within $0.25 \mathrm{D}$ of the targeted refraction and all stable within $0.25 \mathrm{D}$ by last follow-up of up to nine months.

A study by Hengerer et al. ${ }^{18}$ evaluated the LAL in 15 patients with axial hyperopia (with or without toric component) with a follow-up of 12 months, $93 \%$ of the eyes achieved +/-0.5 D of the targeted refractive correction and all except one eye showed $<0.25 \mathrm{D}$ change between the one month post lock-in and the 12 month visit. The same group evaluated the combined correction of sphere and astigmatism in eyes with axial myopia; ${ }^{19}$ the results from this study were also encouraging with 20 (96\%) of 21 eyes within $0.50 \mathrm{D}$ of the intended refractive correction and stable within $0.25 \mathrm{D}, 12$ months after lock-in.

Results from other LAL series ${ }^{20-24}$ have shown similar results and together demonstrate the effectiveness and precision of this technology. It is of interest to mention that there was not a single complication (excluding two cases of slight PCO at 12 months) or adverse event reported in any of the studies previously mentioned.

The largest LAL series was recently published and included 122 eyes with eighteen months of follow-up. In this prospective study, $97 \%$ of patients were within $0.25 \mathrm{D}$ of attempted spherical equivalent refraction and $100 \%$ had an uncorrected visual acuity of $20 / 25$ or better. In this large series, there were three cases of post-operative corneal oedema that resolved completely after the first week (before any UV irradiation of the $L A L$ ), three cases 
of increased intraocular pressure which normalised without the need for topical therapy and two patients that suffered from dry eye syndrome. None of these adverse events were attributed to the LAL or the irradiation protocol.

Only one case of a complication inherent to the LAL has been reported in the literature. ${ }^{25} \mathrm{~A}$ 57-year-old female that, after uneventful phacoemulsification with implantation of a LAL and the first adjustment procedure, returned with a decrease in BCVA to 20/125 and a refraction of $-3.25 \mathrm{D}$. A distinct paracentral elevation in the lens was noted on slit lamp examination. Upon questioning, the patient admitted she had not used the UV protecting glasses at several occasions during the post-operative period and had exposed herself to sunlight for several hours. This case underscores the importance of compliance with post-operative UV protection glasses, something that must be stressed by the surgeon and staff in order to ensure the desired outcome.

An important caveat in patient selection is that a pre-operative dilated pupil size larger than $7.0 \mathrm{~mm}$ must be confirmed before implantation off the LAL, since this is the pupil size needed postoperatively for the adjustments and specially the lock-in procedure.

It is clear from the previous studies that the LAL can be adjusted postoperatively to correct myopia,,$^{11,19}$ hypermetropia $a^{17,18}$ and astigmatism ${ }^{20,21}$ with great precision. The next challenge for this technology is to address presbyopia. ${ }^{14,21}$ For this purpose, the easiest, already available option is monovision. Monovision can be created by adjusting the LAL to the desired amount of myopia, then the patient is given time to adjust to his monovision, which, in case the patient cannot adapt or is not happy, can be further modified or completely reversed with a second adjustment before the lock-in of the lens, something that can only be done with this technology. Another area that is currently under development is a multifocal profile in which a small near zone can be added during a subsequent adjustment, after all sphero-cylindrical error has been corrected, allowing the size, power and location of the add zone to be customised to the patient. Results from a pilot study with this approach, reached uncorrected distance visual acuity of 20/25 in $83.3 \%$ of the eyes and uncorrected near visual acuity of J2 in $87.5 \%$ of the eyes. ${ }^{26}$ Another approach to presbyopia with the LAL that is being pursued, consists of increasing the asphericity of the lens with the LDD to increasing depth of focus. ${ }^{27}$

One area where the LAL can be extremely helpful is in the post-refractive surgery cases, in which inaccuracy with IOL calculation and refractive surprises are common. Data on 16 eyes post-refractive surgery was recently presented by Dr Rocha during the 2011 annual meeting of the Canadian Ophthalmological Society. ${ }^{28}$ Seventy-five per cent of the eyes were within 0.25 D of intended correction and $95 \%$ were within $0.50 \mathrm{D}$, results that seem rather promising.

\section{Discussion}

As cataract surgery has evolved and small incision phacoemulsification has become the dominant procedure, surgeons have been on a continual quest to provide patients with an emmetropic visual outcome. ${ }^{1}$ As visual outcomes have improved over the years with developments in biometry and IOL calculation formulas, ${ }^{29-31}$ patients have become more demanding, expecting at least emmetropia if not presbyopic correction.
With current methods of $\mathrm{IOL}$ power determination, the vast majority of patients achieve a UCVA of 20/40 or better. A much smaller percentage achieves optimal vision without spectacles. ${ }^{1}$ The variables that affect post-operative UCVA include imprecise IOL power determinations, pre-existing corneal astigmatism, surgical induced astigmatism and previous corneal refractive surgery. ${ }^{32-36}$

With the LAL technology, after optimised IOL calculations have been used to achieve the best possible post-operative outcome with the available pre-operative data, biometry and the use of newer and improved formulas, The LAL provides surgeons a means to fine tune refractive power in the post-operative period, thus benefiting from the latest developments in IOL calculations and its unique capability of precise correction of any residual sphero-cylindrical refractive error.

There are mainly two potential shortcomings with this technology, the first one is the number of post-operative visits required for the adjustment and lock-in procedures, something that can be managed by a standardised protocol for the post-operative visits and trained staff, since the LDD treatment itself takes usually under two minutes. The other issue is compliance with the wear of UV protective spectacles by the patient until lock-in is performed to avoid unplanned UV exposure with its consequent change in LAL power (this is because the photoreactive macromer undergoes photopolymerisation when exposed to ambient UV light). This is something that the patient needs to understand and accept before going ahead with the procedure.

We probably have not yet seen the full potential of this technology, the Digital LDD has corrected up to $4 \mathrm{D}$ of refractive error during in vitro studies and its capable of customised adjustments that could be applied to compensate for irregular astigmatism, such as after corneal transplantation, radial keratotomy, or irregular corneal ablations. ${ }^{4}$ Similarly, Wavefront-based adjustments could be made that unlike excimer laser correction of higher order aberrations in the cornea, would not be subject to the unpredictability of wound healing. ${ }^{37}$ As previously mentioned, different approaches are currently under investigation for the management of presbyopia with encouraging results.

The closest development to the LAL in the post-operative adjustable lenses arena, although clearly inferior, is the Acri.Tec AR-1 PC/IOL (now Carl Zeiss, Meditech, Jena Germany), which is a polymethylmethacrylate lens with a $5.5 \mathrm{~mm}$ optic. Connected to the optic is a haptic containing an adjustment element, formed by an interruption of the haptic consisting of a cylinder $1.0 \mathrm{~mm}$ high attached to the optic, which contains a piston attached to the outer part of the haptic. The cylinder and the piston can be moved relative to each other. This element allows the optical part of the IOL to move along the optical axis. Adjustment of the lens is performed by an IOL optic manipulator. The main issues with this lens is that it only allows for spherical adjustments and that it requires a second intraocular procedure two weeks after phacoemulsification and IOL implantation for adjustment. ${ }^{38}$

The LAL technology uses UV light and a photoreactive macromer to induce refractive changes in the $\mathrm{IOL}$, currently another group is exploring the use of the femtosecond laser to alter the refractive 
index of the IOL, a technology called 'refractive index shaping' which is still in the early developmental stages. ${ }^{39}$

The LAL is the only IOL available that allows for post-operative refractive adjustment and is currently available in Europe, Canada and Mexico, while it is still undergoing clinical trials in the US.

\section{Conclusion}

The LAL technology has demonstrated to be a safe, accurate and reliable method of post-operative, non-surgical correction of residual sphero-cylindrical refractive error after cataract surgery and IOL implantation. A clear advantage in achieving emmetropia in standard cases but something that can be invaluable for the increasing number of post-refractive cataract surgery patients.
1. Hengerer FH, Dick HB, Conrad-Hengerer I, Clinica Evaluation of an Ultraviolet Light Adjustable Intraocular Lens Implanted after Cataract Removal Eighteen Months Follow-up, Ophthalmology, 2011;18(12):2382-8.

2. GN K, inventor Cooper Vision Inc., assignee, Small Incision Intraocular Lens with Adjsutable Refractive Power US PATENT 1990.

3. $\mathrm{HC}$ E, inventor Harry C Eggleston, assignee, Adjustable and removable intraocular lens implant, US patent 1997.

4. Schwartz DM, Light-adjustable lens, Trans Am Ophthalmol Soc, 2003;101:417-36

5. Werblin TP, Multicomponent intraocular lens, I Refract Surg, 1996;12(1):187-9.

6. Matthews MW, Eggleston HC, Hilmas GE, Development of a repeatedly adjustable intraocular lens, I Cataract Refract Surg, 2003:29(11):2204-10

7. Matthews MW, Eggleston HC, Pekarek SD, Hilmas GE, Magnetically adjustable intraocular lens, I Cataract Refract surg 2003;29(11):2211-6.

8. Lichtinger A, Sandstedt CA, Padilla K, et al., cornea endothelial safety after ultraviolet light treatment of the light-adjustable intraocular lens, I Cataract Refract Surg, 2011;37(2):324-7

9. Nishi O, Nishi K, Sakanishi K, Inhibition of migrating lens epithelial cells at the capsular bend created by the rectangular optic edge of a posterior chamber intraocular lens, Ophthalmic Surg Lasers, 1998:29(7):587-94.

10. Olson R, Mamalis N, Haugen B, A light adjustable lens with injectable optics, Ophthalmol Clin North Am, 2006:19(1):135-42, vii

11. Chayet A, Sandstedt C, Chang S, et al., Correction of myopia after cataract surgery with a light-adjustable lens, Ophthalmology, 2009;116(8):1432-5.

12. Olson $\mathrm{R}$, Mamalis $\mathrm{N}$, Haugen $\mathrm{B}, \mathrm{A}$ light adjustable lens with injectable optics, Curr Opin Ophthalmol, 2006;17(1):72-9.

13. Schwartz DM, Sandstedt CA, Chang SH, et al., Light-adjustable lens: development of in vitro nomograms, Trans Am Ophthalmol Soc, 2004:102:67-72.

14. Sandstedt CA, Chang SH, Grubbs RH, Schwartz DM, Light-adjustable lens: customizing correction for multifocality and higher-order aberrations, Trans Am Ophthalmol Soc, 2006:104:29-39.

15. Werner L, Yeh O, Haymore J, et al., Corneal endothelial safety with the irradiation system for light-adjustable intraocular lenses, I Cataract Refract Surg, 2007;33(5):873-8.

16. Werner L, Chang W, Haymore J, et al., Retinal safety of the irradiation delivered to light-adjustable intraocular lenses evaluated in a rabbit model, I Cataract Refract Surg, 2010;36(8):1392-7.

17. Chayet A, Sandstedt CA, Chang SH, et al, Correction of residual hyperopia after cataract surgery using the light adjustable intraocular lens technology, Am I Ophthalmol, adjustable intraocular

18. Hengerer FH, Hutz WW, Dick HB, Conrad-Hengerer I, Combined correction of axial hyperopia and astigmatism using the light adjustable intraocular lens, Ophthalmology, 2011;118(7):1236-41.

19. Hengerer FH, Hutz WW, Dick HB, Conrad-Hengerer I, Combined correction of sphere and astigmatism using the light-adjustable intraocular lens in eyes with axial myopia, I Cataract Refract Surg, 2011;37(2):317-23,

20. Lichtinger A, Sandstedt CA, Schwartz DM, Chayet AS, Correction of astigmatism after cataract surgery using the light adjustable lens: a 1-year follow-up pilot study, J Refract Surg, 2011;27(9):639-42.

21. Chayet A, Sandstedt C, Chang S, et al., Use of the light-adjustable lens to correct astigmatism afte cataract surgery, Br I Ophthalmol, 2010;94(6):690-2.

22. Hengerer FH, Conrad-Hengerer I, Buchner SE, Dick HB, Evaluation of the Calhoun Vision UV Light Adjustable Lens implanted following cataract removal, J Refract Surg, 2010:26(10):716-21.

23. Hengerer FH, Conrad-Hengerer I, Hutz WW, Dick B Adjustment of sphero-cylindrical refractive errors in hyperopic eyes: 6-month results after cataract surger and implantation of a light-adjustable intraocular lens, Klin Monbl Augenheilkd, 2010;227(9):729-34.

24. von Mohrenfels CW, Salgado J, Khoramnia R, et al., Clinical results with the light adjustable intraocular lens after cataract surgery, J Refract Surg, 2010;26(5):314-20.

25. Hafezi F, Seiler T, Iseli HP, Light-adjustable lens complication, Ophthalmology, 2010:117(4):848-e1.

26. Chayet A, Presbyopia Correction With Light-Adjustable Lens, Presented at: ASCRS Symposium on Cataract, IOL and Refractive Surgery, San diego, California, 25-29 March 2011
27. Paulig S Binovision, A Novel Approach to Presbyopia Correction, I Cataract Refract Surg, 2011:34-6.

28. Rocha G, The Light Adjustable Lens in Post-Refractive Surgery Cases, Presented at: 2011 Annual meeting of the Canadian Ophthalmological Society, Vancouver, Britsh Columbia, Canada, 9-12 June 2011.

29. Savini G, Barboni P, Carbonelli M, Hoffer KJ, Accuracy of scheimpflug corneal power measurements for intraocular Iens power calculation, I Cataract Refract Surg, lens power calculation

30. Fam HB, Lim KL, A comparative analysis of intraocular lens power calculation methods after myopic excimer laser surgery, J Refract Surg, 2008;24(4):355-60.

31. Haigis W, Lege B, Miller N, Schneider B, Comparison of immersion ultrasound biometry and partial coherence interferometry for intraocular lens calculation according to Haigis, Graefes Arch Clin Exp Ophthalmol, 2000;238(9):765-73.

32. Jin GJ, Crandall AS, Jones JJ, Intraocular lens exchange due to incorrect lens power, Ophthalmology, 2007:114(3):417-24.

33. Haigis $W$, Intraocular lens calculation in extreme myopia, $J$ Cataract Refract Surg, 2009;35(5):906-11.

34. Zuidervaart W, Luyten GP, A retrospective analysis of five intra-ocular lenses and the predictive value of six different intra-ocular lens power calculation formulas, Ophthalmologica, 2005;219(6):390-3.

35. Wang L, Booth MA, Koch DD, Comparison of intraocula Iens power calculation methods in eyes that have undergone LASIK, Ophthalmology, 2004;111(10):1825-31.

36. Packer M, Brown LK, Hoffman RS, Fine $I H$, Intraocular lens power calculation after incisional and thermal keratorefractive surgery, I Cataract Refract surg, 2004;30(7):1430-4

37. Roberts $C$, The cornea is not a piece of plastic, J Refract Surg, 2000;16(4):407-13.

38. Jahn CE, Schopfer DC, Cataract surgery with implantation of a mechanically and reversibly adjustable intraocular lens: *Acri.Tec AR-1 posterior chamber intraocular lens, Arch Ophthalmol, 2007;125(7):936-9.

39. Bille JF, Generation and in situ modification of customized IOLS, Presented at: ASCRS Symposium on Cataract, IOL and Refractive Surgery, San Diego, California, 28 March 2011. 